\title{
SOBOLEV INEQUALITIES FOR WEIGHT SPACES AND SUPERCONTRACTIVITY
}

BY

\section{JAY ROSEN(1)}

ABSTRACT. For $\phi \in C^{2}\left(\mathrm{R}^{n}\right)$ with $\phi(x)=a|x|^{1+s}$ for $|x| \geqslant x_{0}$, $a, s>0$, define the measure $d \mu=\exp (-2 \phi) d^{n} x$ on $\mathrm{R}^{n}$. We show that for any $k \in \mathrm{Z}^{+}$

$$
\begin{aligned}
& \int|f|^{2}|\lg (|f|)|^{2 s k /(s+1)} d \mu \\
& \quad<c\left\{\sum_{|\alpha|=0}^{k}\left\|D^{\alpha}\right\|_{L_{2}(d \mu)}^{2}+\|f\|_{L_{2}(d \mu)}^{2} \cdot\left|\lg \left(\|f\|_{L_{2}(d \mu)}\right)\right|^{2 s k /(s+1)}\right\}
\end{aligned}
$$

As a consequence we prove $e^{-t \nabla^{*} \cdot \nabla}: L_{q}\left(\mathrm{R}^{n}, d \mu\right) \rightarrow L_{p}\left(\mathrm{R}^{n}, d \mu\right), p, q \neq 1$, $\infty$, is bounded for all $t>0$.

1. Introduction. The classical Sobolev inequalities state

$$
\|f\|_{p} \leqslant c \sum_{|\alpha|=k}\left\|D^{\alpha} f\right\|_{q}, \quad f \in C_{0}^{\infty}\left(\mathbf{R}^{n}\right)
$$

where $p=(1 / q-k / n)^{-1}, 1 \leqslant p<\infty, \alpha$ is an $n$-tuple, $\alpha=\left(\alpha_{1}, \ldots, \alpha_{n}\right)$, and $D^{\alpha}=\partial^{\alpha}{ }_{1} / \partial x_{1}^{\alpha_{1}} \cdots \partial^{\alpha} n / \partial x_{n}^{\alpha}[14]$.

Recently, L. Gross has proven a beautiful analogue of the Sobolev inequalities for the Gaussian measure $d \nu=(2 \pi)^{-n / 2} \exp \left(-|x|^{2} / 2\right) d^{n} x$ on $\mathbf{R}^{n}$ [1]. This "logarithmic" Sobolev inequality states

$$
\begin{aligned}
(2 \pi)^{-n / 2} \int|f|^{2} \lg (|f|) \exp \left(-|x|^{2} / 2\right) d^{n} x \\
\leqslant \sum_{i=1}^{n}\left\|\frac{\partial f}{\partial x_{i}}\right\|_{L_{2}(d \nu)}^{2}+\|f\|_{L_{2}(d \nu)}^{2} \cdot \lg \left(\|f\|_{L_{2}(d \nu)}\right) .
\end{aligned}
$$

Furthermore, Gross has exhibited a function $f \in L_{2}(d \nu)$ with $\sum_{i=1}^{n}\left\|\partial f / \partial x_{i}\right\|_{L_{2}}^{2}(d \nu)$ $<\infty$ but

$$
\int|f|^{2} \lg (\mid f i) \lg ^{+}\left(\lg ^{+}(|f|)\right) \exp \left(-|x|^{2} / 2\right) d^{n} x=\infty,
$$

showing how good his inequality (2) is. Similar, higher order inequalities for the

Received by the editors May 14, 1975.

AMS (MOS) subject classifications (1970). Primary 46E35, 46E30, 35 B45.

(1) This paper is based on the author's doctoral dissertation, Princeton University,

1974. The author was supported by NSF grant MPS74 13252. 
Gaussian measure have been proved by G. Feissner [2] .

If $\phi \in C\left(\mathbf{R}^{n}\right)$ with $\int \exp (-2 \phi) d^{n} x<\infty$ let us define the weight space $L_{2}^{(k)}\left(\mathbf{R}^{n}, \phi\right)$ to be the completion of $C_{0}^{\infty}\left(\mathbf{R}^{n}\right)$ in the norm $\Sigma_{|\alpha|=0}^{k}\left\|D^{\alpha} f\right\|_{2, \phi}$, where

$$
\|g\|_{2, \phi}^{2}=\frac{\int|g|^{2} \exp (-2 \phi) d^{n} x}{\int \exp (-2 \phi) d^{n} x} .
$$

The main aim of this paper is to develop a method for obtaining precise Sobolev inequalities for a large class of weights $\phi$.

To illustrate our results assume $\phi \in C^{2}\left(R^{n}\right)$, with $\phi=a|x|^{1+s}$ for large $|x| \geqslant x_{0} ; a>0, s>0$. We will show that

$$
\begin{aligned}
& \int_{\mathbf{R}^{n}}|f|^{2}|\lg (\mid f i)|^{2 k s /(s+1)} \exp (-2 \phi) d^{n} x \\
& \quad \leqslant c\left\{\sum_{|\alpha|=0}^{k}\left\|D^{\alpha} f\right\|_{2, \phi}^{2}+\|f\|_{2, \phi}^{2}\left|\lg \left(\|f\|_{2, \phi}\right)\right|^{2 k s /(s+1)}\right\}, \\
& \quad f \in L_{2}^{(k)}\left(\mathbf{R}^{n}, \phi\right) .
\end{aligned}
$$

This result is best possible in the sense that for any $m \in \mathbf{Z}^{+}$we exhibit $f \in$ $L_{2}^{(k)}\left(\mathbf{R}^{n}, \phi\right)$ with

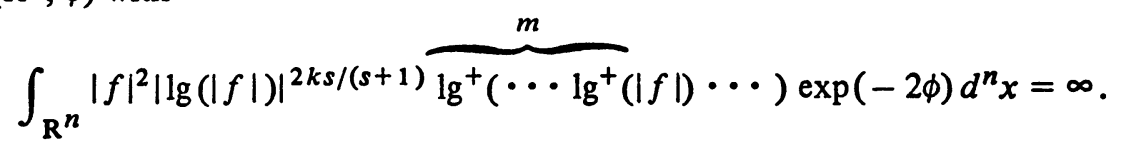

L. Gross has also shown [1] how 'logarithmic' Sobolev inequalities can be used to prove that $e^{-t \nabla^{*} \cdot \nabla}, t>0$, is a hypercontractive semigroup. Recall that a selfadjoint contraction semigroup $e^{-t F}$ on a probability space $(M, d \mu)$ is called hypercontractive if $e^{-t F}: L_{q} \rightarrow L_{p}$ is bounded for $p, q \neq 1, \infty$ and $t \geqslant t(p, q)$ [3]. In particular E. Nelson has shown [4] that for the Gaussian measure $d v=$ $(2 \pi)^{-n / 2} \exp \left(-|x|^{2} / 2\right) d^{n} x$ on $\mathrm{R}^{n}$,

$$
e^{-t \nabla^{*} \cdot \nabla}: L_{q}\left(\mathbf{R}^{n}, d \nu\right) \rightarrow L_{p}\left(\mathbf{R}^{n}, d \nu\right)
$$

is bounded, $p, q \neq 1, \infty$, only if $t \geqslant \lg \left([(p-1) /(q-1)]^{1 / 2}\right)$, in which case it is a contraction. Using our precise Sobolev inequalities, together with Gross's theorem, we show that for a large class of weights $\phi$,

$$
e^{-t \nabla^{*} \cdot \nabla}: L_{q}\left(\mathbf{R}^{n}, \phi\right) \rightarrow L_{p}\left(\mathbf{R}^{n}, \phi\right)
$$

$p, q \neq 1, \infty$, is bounded for all $t>0$ ! We call this property of the semigroup $e^{-t \nabla^{*} \cdot \nabla}$ supercontractivity.

We note that J.-P. Eckmann [5] has independently extended Gross's methods 
to prove that $e^{-t \nabla^{*} \cdot \nabla}$ is hypercontractive for many weights $\phi$. We have been able to push his technique to prove supercontractivity, but it is not powerful enough to prove our precise Sobolev inequalities.

In $\S \S 2$ and 3 we prove our basic Sobolev inequalities. Supercontractivity is proven in $\S 4$. In $\S 5$ we describe some weights which satisfy the general requirements of our theorems, and we show that in many cases our results are best possible.

We remark that our inequalities have also been used to determine the fine fluctuations of paths in the $P(\phi)_{1}$ Markoff processes [9].

AcKNowledgements. I would like to thank my thesis advisor, Professor Barry Simon, for suggesting the sort of problems discussed in this paper, and for streamlining my original proof of Theorem 1. Professors Edward Nelson and Abel Klein have made helpful suggestions.

2. First order inequalities. Throughout this paper we assume $\phi \in C^{2}\left(R^{n}\right)$ with $\int \exp (-2 \phi) d^{n} x<\infty$.

THEOREM 1. Let $r>0$ be such that

$$
|\phi(x)|^{r} \leqslant a(\nabla \phi \cdot \nabla \phi-\Delta \phi+b)
$$

then

$$
\begin{aligned}
& \int|f|^{2}|\lg (|f|)|^{r} \exp (-2 \phi) d^{n} x \\
& \leqslant c\left\{\sum_{i=1}^{n}\left\|\frac{\partial f}{\partial x_{i}}\right\|_{2, \phi}^{2}+\|f\|_{2, \phi}^{2}+\|f\|_{2, \phi}^{2} \cdot \|\left.\lg \left(\|f\|_{2, \phi}\right)\right|^{r}\right\} \\
& \quad f \in L_{2}^{(1)}\left(\mathbf{R}^{n}, \phi\right) .
\end{aligned}
$$

If, in addition, $r \geqslant 1$, then

$$
\begin{aligned}
& \int|f|^{2} \lg (|f|) \exp (-2 \phi) d^{n} x \\
& \leqslant c\left(f,\left(\nabla^{*} \cdot \nabla+1\right)^{1 / r} f\right)+\|f\|_{2, \phi}^{2} \lg \left(\|f\|_{2, \phi}\right), \\
& \quad f \in Q\left(\left(\nabla^{*} \cdot \nabla\right)^{1 / r}\right)
\end{aligned}
$$

and

$$
\int|f|^{2} \lg (|f|) \exp (-2 \phi) d^{n} x \leqslant c\left(\sum_{i=1}^{n}\left\|\frac{\partial f}{\partial x_{i}}\right\|_{2, \phi}^{2 / r}+1\right)
$$

$$
f \in L_{2}^{(1)}\left(\mathbf{R}^{n}, \phi\right),\|f\|_{2, \phi}=1 .
$$


Proof. We may assume $\int \exp (-2 \phi) d^{n} x=1$. We will first prove our theorem for all $f$ such that $\|f\|_{2, \phi}=1$. For such an $f$ we have

$$
\int \exp \left(\lg ^{+}\left(|f|^{2}\right)\right) \exp (-2 \phi) d^{n} x \leqslant 2 .
$$

Setting $h=\left(\lg ^{+}\left(|f|^{2}\right)\right)^{r} \geqslant 0$ we can write this as

$$
\int \exp \left(h^{1 / r}-2 \phi\right) d^{n} x \leqslant 2
$$

Let

$$
U=\left\{x \in \mathbf{R}^{n} \mid h^{1 / r}-2 \phi \leqslant 0\right\}, \quad V=U^{c}=\left\{x \in \mathbf{R}^{n} \mid h^{1 / r}-2 \phi>0\right\} .
$$

Since $\exp (\cdot) \geqslant 0,(10)$ implies

$$
\int_{V} \exp \left(h^{1 / r}-2 \phi\right) d^{n} x \leqslant 2
$$

Since, by the definition of $V, h_{\mid V}^{1 / r}-2 \phi_{\mid V} \geqslant 0$, (11) tells us that $\left[h_{\mid V}^{1 / r}-2 \phi_{\mid V}\right]$ $\in \bigcap L_{p}\left(\mathbf{R}^{n}, d^{n} x\right)$ and $\int_{V} 1 d^{n} x<2$, hence

$$
\left[h_{\mid V}^{1 / r}-2 \phi_{\mid V}\right]^{r} \in \bigcap L_{p}\left(R^{n}, d^{n} x\right)
$$

Now, the classical Sobolev inequality (1) implies [8] that $f \leqslant d\|f\|_{n}(-\Delta+1)$ as forms on $L_{2}\left(\mathbf{R}^{n}, d^{n} x\right)$ for all $f \in L_{n}\left(\mathbf{R}^{n}, d^{n} x\right)$, so that (12) implies

$$
\left[h_{\mid V}^{1 / r}-2 \phi_{\mid V}\right]^{r} \leqslant k(-\Delta+1)
$$

If $r \geqslant 1$, the convexity and monotonicity of $x^{r}$ now give

$$
\begin{aligned}
h_{\mid V} & =\left(h_{\mid V}^{1 / r}\right)^{r} \leqslant\left[h_{\mid V}^{1 / r}-2 \phi_{\mid V}+2\left|\phi_{\mid V}\right|\right]^{r} \\
& \leqslant 2^{r-1}\left\{\left[h_{\mid V}^{1 / r}-2 \phi_{\mid V}\right]^{r}+\left(2\left|\phi_{\mid V}\right|\right)^{r}\right\} \leqslant k\left(-\Delta+\left|\phi_{\mid V}\right|^{r}+1\right) .
\end{aligned}
$$

A similar argument works for $0<r \leqslant 1$, using the monotonicity and subadditivity of $x^{r}$.

Since the definition of $U$ requires

$$
h_{\mid U} \leqslant\left(2\left|\phi_{\mid U}\right|\right)^{r},
$$

we have, combining (13) and (14),

$$
\left(\lg ^{+}\left(|f|^{2}\right)\right)^{r}=h \leqslant k\left(-\Delta+|\phi(x)|^{r}+1\right) .
$$

Then by our hypothesis (5)

$$
\left(\lg ^{+}\left(|f|^{2}\right)\right)^{r} \leqslant k(-\Delta+\nabla \phi \cdot \nabla \phi-\Delta \phi+1)
$$


as forms on $L_{\mathbf{2}}\left(\mathbf{R}^{n}, d^{n} x\right)$, where $k$ is independent of $f$, if $\|f\|_{2, \phi}=1$.

Now, multiplication by $\exp (-\phi)$ is a unitary equivalence from $L_{2}\left(\mathbf{R}^{n}, \phi\right)$ to $L_{2}\left(\mathbf{R}^{n}, d^{n} x\right)$, which takes $\nabla^{*} \cdot \nabla$ into

$$
\exp (-\phi)\left(\nabla^{*} \cdot \nabla\right) \exp (\phi)=-\Delta+\nabla \phi \cdot \nabla \phi-\Delta \phi
$$

so that (16) is equivalent to

$$
\left(\lg ^{+}\left(|f|^{2}\right)\right)^{r} \leqslant k\left(\nabla^{*} \cdot \nabla+1\right)
$$

as forms on $L_{2}\left(\mathbf{R}^{n}, \phi\right)$.

In particular (17) gives

$$
\int|f|^{2}\left(\lg ^{+}\left(|f|^{2}\right)\right)^{r} \exp (-2 \phi) d^{n} x \leqslant k\left(\left(f, \nabla^{*} \cdot \nabla f\right)+1\right)
$$

which implies (6) for $\|f\|_{2, \phi}=1$.

Furthermore, if $r \geqslant 1$ we may use Loewner's theorem [10], which tells us that for $r \geqslant 1$ the $r$ th root is a monotone operator function. (17) then implies

$$
\lg \left(|f|^{2}\right) \leqslant \lg ^{+}\left(|f|^{2}\right) \leqslant k\left(\nabla^{*} \cdot \nabla+1\right)^{1 / r}
$$

which, as before, yields (7) for $\|f\|_{2, \phi}=1$.

(6) and (7) now follow for all $f$ from the following lemma.

LEMMA 2. Let $d \mu$ be an arbitrary probability measure and let $F$ be an operator on $L_{2}(d \mu)$ with

$$
\int|f|^{2}|\lg (|f|)|^{r} d \mu \leqslant\|F f\|_{2}^{2}
$$

for all $f \in D(F),\|f\|_{2}=1$. If $r \geqslant 1$, then for any $p, q \neq 1, \infty, 1 / p+1 / q=1$, we have

$$
\int|f|^{2}|\lg (|f|)|^{r} d \mu \leqslant q^{r-1}\|F f\|_{2}^{2}+p^{r-1}\|f\|_{2}^{2}\left|\lg \left(\|f\|_{2}\right)\right|^{r}, \quad \text { all } f \in D(F) .
$$

If $0<r \leqslant 1$, then

$$
\int|f|^{2}|\lg (|f|)|^{r} d \mu \leqslant\|F f\|_{2}^{2}+\|f\|_{2}^{2}\left|\lg \left(\|f\|_{2}\right)\right|^{r}, \quad \text { all } f \in D(F) .
$$

and if $r=1$ the inequality $\int|f|^{2} \lg (|f|) d \mu \leqslant\|F f\|_{2}^{2}, f \in D(F),\|f\|_{2}=1$, implies

$$
\int|f|^{2} \lg (|f|) d \mu \leqslant\|F f\|_{2}^{2}+\|f\|_{2}^{2} \lg \left(\|f\|_{2}\right), \text { all } f \in D(F) .
$$

Proof. Consider first the case $r \geqslant 1$. Take $f \in D(F)$. By assumption

$$
\int|f|^{2}\left|\lg \left(|f| /\|f\|_{2}\right)\right|^{r} d \mu \leqslant\|F f\|_{2}^{2} .
$$


By convexity and monotonicity of $x^{r}$, for any $p, q \neq 1, \infty, 1 / p+1 / q=1$, we have

$$
\begin{aligned}
\int|f|^{2}|\lg (|f|)|^{r} d \mu & =\int|f|^{2}\left|\lg \left(|f| /\|f\|_{2}\right)+\lg \left(\|f\|_{2}\right)\right|^{r} d \mu \\
& =\int|f|^{2}\left|\frac{q \lg \left(|f| /\|f\|_{2}\right)}{q}+\frac{p \lg \left(\|f\|_{2}\right)}{p}\right|^{r} d \mu \\
& \leqslant \int|f|^{2}\left(\frac{q\left|\lg \left(|f| /\|f\|_{2}\right)\right|}{q}+\frac{\left|p \lg \left(\|f\|_{2}\right)\right|}{p}\right)^{r} d \mu \\
& \leqslant q^{r-1} \int|f|^{2}\left|\lg \left(|f| /\|f\|_{2}\right)\right|^{r} d \mu+p^{r-1}\|f\|_{2}^{2}\left|\lg \left(\|f\|_{2}\right)\right|^{r} \\
& \leqslant q^{r-1}\|F f\|_{2}^{2}+p^{r-1}\|f\|_{2}^{2}\left|\lg \left(\|f\|_{2}\right)\right|^{r}
\end{aligned}
$$

The assertion for $0<r \leqslant 1$ follows similarly using the monotonicity and subadditivity of $x^{r}$. The assertion for $r=1$ is trivial.

Finally, (8) follows from (7) for $f$ normalized by the spectral theorem and Holder's inequality.

\section{Higher order inequalities.}

THEOREM 3. Let $r>0$ be such that $|\phi(x)|^{r} \leqslant a(\nabla \phi \cdot \nabla \phi-\Delta \phi+b)$; then for all $k \in \mathrm{N}$

$$
\begin{aligned}
& \int|f|^{2}|\lg (|f|)|^{r k} \exp (-2 \phi) d^{n} x \\
& \leqslant c\left\{\sum_{|\alpha|=0}^{k}\left\|D^{\alpha} f\right\|_{2, \phi}^{2}+\|f\|_{2, \phi}^{2} \|\left.\lg \left(\|f\|_{2, \phi}\right)\right|^{r k}\right\},
\end{aligned}
$$

$f \in L_{2}^{(k)}\left(\mathbf{R}^{n}, \phi\right)$

Proof. Let us prove (19) by induction on $k$. The case $k=1$ is our first order inequality (6). Assume we have proven (19) for $k=1, \ldots, m$. Let us show that

$$
\int|f|^{2}|\lg (|f|)|^{r(m+1)} \exp (-2 \phi) d^{n} x \leqslant c\left(1+\sum_{|\alpha|=0}^{m+1}\left\|D^{\alpha} f\right\|_{2, \phi}\right)^{5},
$$

$$
f \in L_{2}^{(m+1)}\left(\mathbf{R}^{n}, \phi\right)
$$

Then, by homogeneity, and our usual use of monotonicity, convexity and subadditivity

$$
\begin{aligned}
& \int|f|^{2}|\lg (|f|)|^{r(m+1)} \exp (-2 \phi) d^{n} x \\
& \leqslant c\left(\sum_{|\alpha|=0}^{m+1}\left\|D^{\alpha} f\right\|_{2, \phi}^{2}+\|f\|_{2, \phi}^{2} \cdot\left|\lg \left(\sum_{|\alpha|=0}^{m+1}\left\|D^{\alpha} f\right\|_{2, \phi}\right)\right| r(m+1)\right.
\end{aligned}
$$


Then, since $(\lg (x))^{r(m+1)} \leqslant b x, x \geqslant 1$, for some $b,(21)$ yields (19) for the special case $\|f\|_{2, \phi}=1$. The general case now follows by Lemma 2 .

It suffices by continuity to prove (20) for $f \in C_{0}^{\infty}\left(\mathbf{R}^{n}\right)$. We have

$$
\begin{aligned}
\int f^{2}\left|\lg \left(f^{2}\right)\right|^{r(m+1)} \exp (-2 \phi) d^{n} x \\
\leqslant \int\left(f^{2}+4\right)\left(\lg \left(f^{2}+4\right)\right)^{r(m+1)} \exp (-2 \phi) d^{n} x \\
\leqslant \int\left(f^{2}+4\right)\left(\lg \left(f^{2}+4\right)\right)^{r m} \\
\quad \cdot\left(\lg \left[\left(f^{2}+4\right)\left(\lg \left(f^{2}+4\right)\right)^{r m}\right]\right)^{r} \exp (-2 \phi) d^{n} x .
\end{aligned}
$$

If we set $g=\left(f^{2}+4\right)^{1 / 2}\left(\lg \left(f^{2}+4\right)\right)^{r m / 2}$ we can write (22) as

$$
\begin{gathered}
\int f^{2}\left|\lg \left(f^{2}\right)\right|^{r(m+1)} \exp (-2 \phi) d^{n} x \leqslant \int g^{2}\left(\lg \left(g^{2}\right)\right)^{r} \exp (-2 \phi) d^{n} x \\
\leqslant c\left\{\sum_{i=1}^{n}\left\|\frac{\partial g}{\partial x_{i}}\right\|_{2, \phi}^{2}+\|g\|_{2, \phi}^{2}\left|\lg \left(\|g\|_{2, \phi}\right)\right|^{r}+\|g\|_{2, \phi}^{2}\right\}
\end{gathered}
$$

where the last line follows from our first order inequality (6).

Now

$$
\begin{aligned}
\frac{\partial g}{\partial x_{i}} & =\frac{\partial}{\partial x_{i}}\left(\left(f^{2}+4\right)^{1 / 2}\left(\lg \left(f^{2}+4\right)\right)^{r m / 2}\right) \\
& =\frac{\partial f}{\partial x_{i}} \frac{f}{\left(f^{2}+4\right)^{1 / 2}}\left(\left(\lg \left(f^{2}+4\right)\right)^{r m / 2}+r m\left(\lg \left(f^{2}+4\right)\right)^{r m / 2-1}\right) .
\end{aligned}
$$

Therefore

$$
\left\|\frac{\partial g}{\partial x_{i}}\right\|_{2, \phi}^{2} \leqslant c \int\left|\frac{\partial f}{\partial x_{i}}\right|^{2}\left(\lg \left(f^{2}+4\right)\right)^{r m} \exp (-2 \phi) d^{n} x .
$$

Now, Young's inequality [6], [7] states that

$$
\int|U||V| d \mu \leqslant c\left\{1+\int|U||\lg (|U|)|^{r m} d \mu+\int \exp \left(|V|^{1 / r m}\right) d \mu\right\}
$$

so that, by our induction hypothesis (19),

$$
\begin{aligned}
\left\|\frac{\partial g}{\partial x_{i}}\right\|_{2, \phi}^{2} \leqslant c\left\{1+\left.\int\left|\frac{\partial f}{\partial x_{i}}\right|\right|^{2} \mid \lg \left(\left|\frac{\partial f}{\partial x_{i}}\right|^{2}\right)^{r m} \exp (-2 \phi) d^{n} x\right. \\
\left.+\int \exp \left(\lg \left(f^{2}+4\right)\right) \exp (-2 \phi) d^{n} x\right\}
\end{aligned}
$$

$$
\begin{aligned}
& \leqslant c\left\{1+\sum_{|\alpha|=1}^{m+1}\left\|D^{\alpha} f\right\|_{2, \phi}^{2}+\left\|\frac{\partial f}{\partial x_{i}}\right\|_{2, \phi}^{2} \cdot\left|\lg \left(\left\|\frac{\partial f}{\partial x_{i}}\right\|_{2, \phi}\right)\right|^{r m}+\|f\|_{2, \phi}^{2}\right\} \\
& \leqslant c\left(1+\sum_{|\alpha|=0}^{m+1}\left\|D^{\alpha} f\right\|_{2, \phi}^{2}\right)^{2} .
\end{aligned}
$$


Similarly we see that

$$
\begin{aligned}
\|g\|_{2, \phi}^{2} & =\int\left(f^{2}+4\right)\left(\lg \left(f^{2}+4\right)\right)^{r m} \exp (-2 \phi) d^{n} x \\
& \leqslant c\left(1+\sum_{|\alpha|=0}^{m}\left\|D^{\alpha} f\right\|_{2, \phi}^{2}\right)^{2} .
\end{aligned}
$$

(23), (24) and (25) now prove (20), completing our proof of Theorem 3.

4. Supercontractivity.

THEOREM 4. Let $r>1$ be such that $|\phi(x)|^{r} \leqslant a(\nabla \phi \cdot \nabla \phi-\Delta \phi+b)$; then $e^{-t \nabla^{*} \cdot \nabla}$ is a bounded map from $L_{q}\left(\mathbf{R}^{n}, \phi\right)$ to $L_{p}\left(\mathbf{R}^{n}, \phi\right)$ for any $q, p \neq 1, \infty$, for all $t>0$.

Proof. To prove our theorem we appeal to a result due to L. Gross [1], in a generalized form of J.-P. Eckmann [5].

"Let $\mu$ be a probability measure on $\mathbf{R}^{n}$ and let $G$ be a selfadjoint operator on $L_{2}(d \mu)$. Suppose that the set $C_{B}^{2}$ of twice continuously differentiable functions with bounded first and second derivatives is a core for $G$ and that $\int \bar{f}(G g) d \mu$ $=\int \nabla \bar{f} \cdot \nabla g d \mu, f, g \in C_{R}^{2}$. If there exist constants $0<u$ and $v<\infty$ such that

$$
\int|f|^{2} \lg (|f|) d \mu \leqslant u(f, G f)+v\|f\|_{2}^{2}+\|f\|_{2}^{2} \lg \left(\|f\|_{2}\right),
$$

then $\left\|e^{-t G}\right\|_{q, 1+(q-1) e^{2 t / u}, d \mu} \leqslant e^{t v} . "$

Now Theorem 1 tells us that, with

$$
\begin{gathered}
d \mu=\exp (-2 \phi) d^{n} x / \int \exp (-2 \phi) d^{n} x \\
\int|f|^{2} \lg (|f|) d \mu \leqslant c\left(f,\left(\nabla^{*} \cdot \nabla+1\right)^{1 / r} f\right)+\|f\|_{2}^{2} \cdot \lg \left(\|f\|_{2}\right) .
\end{gathered}
$$

By the spectral theorem this implies that for any $\epsilon>0$ there exists a $c(\epsilon)$ such that

$$
\int|f|^{2} \lg (|f|) d \mu \leqslant \epsilon\left(f, \nabla^{*} \cdot \nabla f\right)+c(\epsilon)\|f\|_{2}^{2}+\|f\|_{2}^{2} \cdot \lg \left(\|f\|_{2}\right) .
$$

The general assertion of our theorem follows from the result quoted.

\section{Applications.}

TheOREM 5. If $\phi \sim a|x|^{1+s}, s>0, a>0$, and $D^{\alpha} \phi \sim a D^{\alpha}|x|^{1+s},|\alpha|=$ 1,2 , as $|x| \rightarrow \infty$, then for any $k \in \mathbf{Z}^{+}$

$$
\begin{aligned}
\int|f|^{2}|\lg (|f|)|^{2 s k /(s+1)} \exp (-2 \phi) d^{n} x \\
\leqslant c\left\{\sum_{|\alpha|=0}^{k}\left\|D^{\alpha} f\right\|_{2, \phi}^{2}+\|f\|_{2, \phi}^{2}\left|\lg \left(\|f\|_{2, \phi}\right)\right|^{2 s k /(s+1)}\right\},
\end{aligned}
$$


$f \in L_{2}^{(k)}\left(\mathbf{R}^{n}, \phi\right)$. If $s>1$, then

$$
\begin{array}{r}
\int|f|^{2} \lg (|f|) \exp (-2 \phi) d^{n} x \leqslant c\left\{\sum_{i=1}^{n}\left\|\frac{\partial f}{\partial x_{i}}\right\|_{2, \phi}^{(s+1) / s}+1\right\}, \\
f \in L_{2}^{(1)}\left(\mathrm{R}^{n}, \phi\right),\|f\|_{2, \phi}=1,
\end{array}
$$

and

$$
e^{-t \nabla^{*} \cdot \nabla}: L_{q}\left(\mathbf{R}^{n}, \phi\right) \rightarrow L_{p}\left(\mathbf{R}^{n}, \phi\right), \quad p, q \neq 1, \infty,
$$

is bounded for all $t>0$. Furthermore, for any $s>0$, if

$$
D^{\alpha} \phi \sim a D^{\alpha}|x|^{1+s}, \quad|\alpha|=0,1, \ldots, k,
$$

then for any $m \in \mathbf{Z}^{+}$, there are $f \in L_{2}^{(k)}\left(\mathbf{R}^{n}, \phi\right)$ with

$$
\left.\int\left|f i^{2}\right| \lg (|f|)\right|^{2 s k /(s+1)} \frac{m}{\lg ^{+}\left(\cdots \cdot \lg ^{+}(|f|) \cdots\right) \exp (-2 \phi) d^{n} x=\infty .}
$$

Proof. (26), (27), and (28) follow from Theorems 1, 3 and 4 once we have verified $|\phi(x)|^{2 s /(s+1)} \leqslant a(\nabla \phi \cdot \nabla \phi-\Delta \phi+b)$, but by our hypothesis both $|\phi(x)|^{2 s /(s+1)}$ and $\nabla \phi \cdot \nabla \phi-\Delta \phi$ are $\sim O\left(|x|^{2 s}\right)$.

To prove the second part of our theorem, consider a function $f$ such that

$$
f(x)=\frac{\exp (\phi(x))|x|^{-(n-1) / 2}}{|x|^{s k}\left(|x| \lg (|x|) \cdots \lg _{m-2}(|x|)\left[\lg _{m-1}(|x|)\right]^{2}\right)^{1 / 2}}
$$

for $|x| \geqslant x_{0}$ large, $f(x)=0$ for $|x| \leqslant x_{0}-1$ and $f(x) \in C^{\infty}\left(\mathrm{R}^{n}\right)$, where we have used the notation $\lg _{j}(x)$ to mean that $\lg (\cdots \lg (x) \cdots)$ occurs $j$ times.

To see that $f \in L_{2}^{(k)}\left(\mathbf{R}^{n}, \phi\right)$ compute for $\alpha=\left(\alpha_{1}, \ldots, \alpha_{n}\right),|\alpha|=k$,

$$
D^{\alpha} f=\frac{\Pi_{i=1}^{n}\left(\partial \phi(x) / \partial x_{i}\right)^{\alpha} \exp (\phi(x))|x|^{-(n-1) / 2}}{|x|^{s k}\left(|x| \lg (|x|) \cdots \lg _{m-2}(|x|)\left[\lg _{m-1}(|x|)\right]^{2}\right)^{1 / 2}}
$$

+ terms smaller at $\infty$ using our assumption (29), and in fact, by (29),

$$
\begin{aligned}
\left\|D^{\alpha} f\right\|_{2, \phi}^{2} & \leqslant c_{0}+c \int_{x_{0}}^{\infty} \frac{d x}{x \lg (x) \cdots \lg _{m-2}(x)\left[\lg _{m-1}(x)\right]^{2}} \\
& =c_{0}+c \int_{x_{0}}^{\infty} \frac{d}{d x}\left(\frac{-1}{\lg _{m-1}(x)}\right) d x=c_{0}+c\left(\lg _{m-1}\left(x_{0}\right)\right)^{-1}<\infty .
\end{aligned}
$$

On the other hand, since $|\phi(x)| \sim O\left(|x|^{s+1}\right)$ 


$$
\begin{aligned}
\int|f|^{2}|\lg (f)|^{2 s k /(s+1)} \lg _{m}^{+}(f) \exp (-2 \phi) d^{n} x \\
\quad \geqslant c_{0} \int_{|x| \geqslant x_{0}} \frac{|\phi(x)|^{2 s k /(s+1)} \lg _{m-1}(\phi(x))|x|^{-(n-1)} d^{n} x}{|x|^{2 s k}|x| \lg (|x|) \cdots \lg _{m-2}(|x|)\left[\lg _{m-1}(|x|)\right]^{2}} \\
\quad \geqslant c_{0} \int_{x_{0}}^{\infty} \frac{1}{x \lg (x) \cdots \lg _{m-1}(x)} d x \\
\quad=c_{0} \int_{x_{0}}^{\infty} \frac{d}{d x}\left(\lg _{m}(x)\right)=\infty .
\end{aligned}
$$

REMARK. Let $P(x)=\sum_{i=0}^{2 p} a_{i} x^{i}$ with $a_{2 p}>0$, and consider the anharmonic oscillator $H$ in $L_{2}\left(\mathbf{R}^{1}, d x\right)$

$$
H=-d^{2} / d x^{2}+P(x) .
$$

The normalized groundstate $\Omega(x)$ is strictly positive and can be written as $\Omega(x)=$ $\exp (-\phi)$, for $\phi$ satisfying all the requirements of Theorem 5 with $s=p$ [7], [11], [12].

For extensions to anharmonic oscillators in $L_{2}\left(\mathbb{R}^{n}, d^{n} x\right)$ see [13].

\section{REFERENCES}

1. L. Gross, Logarithmic Sobolev inequalities, Amer. J. Math. (to appear).

2. G. Feissner, A Gaussian measure analogue to Sobolev's inequality, Thesis, Cornell University, Ithaca, N. Y., 1972.

3. A. Hфegh-Krohn and B. Simon, Hypercontractive semigroups and two dimensional self-coupled Bose fields, J. Functional Analysis 9 (1972), 121-180. MR 45 \#2528.

4. E. Nelson, The free Markoff field, J. Functional Analysis 12 (1973), 211-227. MR 49 \#8556.

5. J.-P. Eckmann, Hypercontractivity for anharmonic oscillators, J. Functional Analysis 16 (1974), 388-404.

6. M. A. Krasnosel'skiî and Ja. B. Rutickir, Convex functions and Orlicz spaces, GITTL, Moscow, 1958; English transl., Noordhoff, Groningen, 1961, pp. 37, 38, 67. MR 21 \#5144; 23 \#A4016.

7. J. Rosen, Logarithmic Sobolev inequalities and supercontractivity for anharmonic oscillators, Thesis, Princeton University, 1974.

8. T. Kato, Perturbation theory for linear operators, Die Grundlehren der math. Wissenschaften, Band 132, Springer-Verlag, New York, 1966, pp. 301-302. MR 34 \#3324.

9. J. Rosen and B.: Simon, Fluctuations in $P(\phi)_{1}$ processes, Ann. Probability 4 (1976).

10. C. Loewner, Uber konvexe Matrixfunctionen, Math. Z. 38 (1934), 177-216.

11. P.-F. Hsieh and Y. Sibuya, On the asymptotic integration of second order linear ordinary differential equations with polynomial coefficients, J. Math. Anal. Appl. 16 (1966), 84-103. MR 34 \#403.

12. B. Simon, Coupling constant analyticity for the anharmonic oscillator, Ann. Physics 58 (1970), 76-136 (Appendix by A. Dicke).

13. - Pointwise bounds on eigenfunctions and wave packets in N-body quantum systems. III, Trans. Amer. Math. Soc. 208 (1975), 317-329.

14. L. Nirenberg, On elliptic partial differential equations, Ann. Scuola Norm. Sup. Pisa (3) 13 (1959), 115-162. MR 22 \#823.

DEPARTMENT OF MATHEMATICS, ROCKEFELLER UNIVERSITY, NEW YORK, NEW YORK 10021 